\title{
Crystal structure of aqua-((E)-N-(2-(2-hydroxybenzylideneamino)ethyl)- 4-methylbenzenesulfonamide)-copper(II), $\mathrm{Cu}\left(\mathrm{H}_{2} \mathrm{O}\right)\left(\mathrm{C}_{16} \mathrm{H}_{17} \mathrm{~N}_{2} \mathrm{O}_{3} \mathrm{~S}\right)$
}

\author{
Shu-Ni Li*,III , Xiao-Yun Wei ${ }^{\mathrm{II}}$, Man-Cheng $\mathrm{Hu}^{\mathrm{II}}$, Jun $\mathrm{Li}^{\mathrm{I}}$ and Feng-Xing Zhang ${ }^{\mathrm{I}}$ \\ I Northwest University, Department of Chemistry, Xi'an 710069, P. R. China \\ II Shaanxi Normal University, School of Chemistry and Materials Science, Xi'an 710062, P. R. China
}

Received November 29, 2006, accepted and available on-line April 17, 2007; CCDC no. 1267/1944
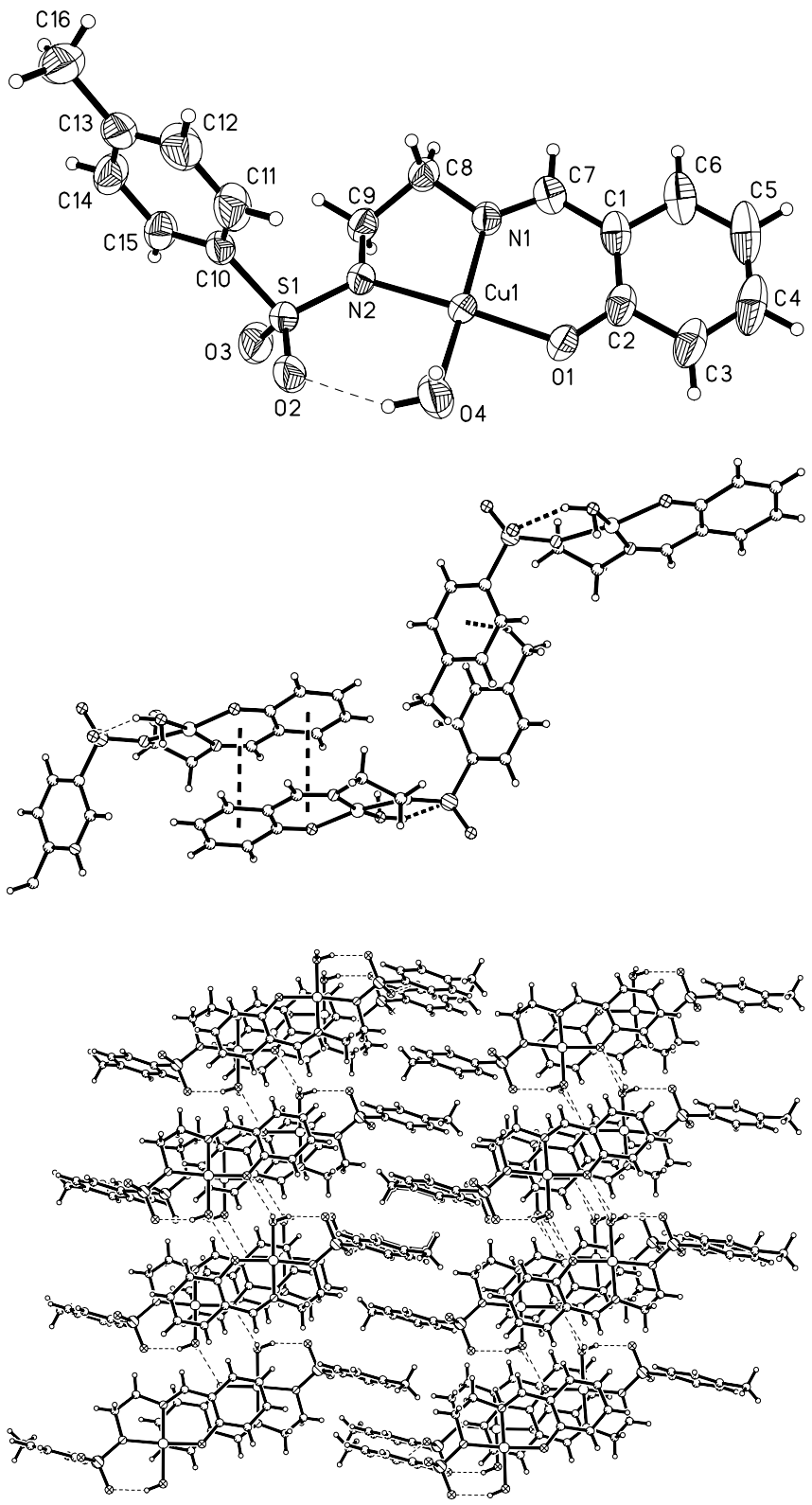

\begin{abstract}
$\mathrm{C}_{16} \mathrm{H}_{18} \mathrm{CuN}_{2} \mathrm{O}_{4} \mathrm{~S}$, triclinic, $P \overline{1}$ (no. 2), $a=7.4366(5) \AA$, $b=7.5339(4) \AA, c=16.617(1) \AA, \alpha=84.597(1)^{\circ}$, $\beta=79.284(1)^{\circ}, \gamma=62.564(3)^{\circ}, V=811.8 \AA^{3}, Z=2$, $R_{\mathrm{gt}}(F)=0.034, w R_{\mathrm{ref}}\left(F^{2}\right)=0.095, T=296 \mathrm{~K}$.
\end{abstract}

\footnotetext{
* Correspondence author (e-mail: lishuni@snnu.edu.cn)
}

\section{Source of material}

The sulfonamide Schiff base ligand (E)- $N$-(2-(2-hydroxybenzylideneamino)ethyl)-4-methylbenzenesulfonamide $(L)$ was synthesized by reacting tosyl-ethylenediamine with one equivalent of salicylaldehyde in ethanol. It is formed as yellow crystals (m.p. 126- $127^{\circ} \mathrm{C}$ ). To $20 \mathrm{~mL}$ hot ethanol solution containing $0.214 \mathrm{~g}$ $(1 \mathrm{mmol}) L, 0.199 \mathrm{~g} \mathrm{Cu}\left(\mathrm{CH}_{3} \mathrm{COO}\right)_{2} \cdot \mathrm{H}_{2} \mathrm{O}(1 \mathrm{mmol})$ in $10 \mathrm{~mL}$ ethanol was added under stirring for $1 \mathrm{~h}$. A dark green solution was formed and filtrated. The filtrate was left for slow evaporation. Dark green block-shaped crystals of the title compound were obtained after 2 weeks.

\section{Experimental details}

The $\mathrm{H}$ atoms were located in difference maps. While the water $\mathrm{H}$ atom positions were refined, the other $\mathrm{H}$ atoms were fixed in idealized positions and refined as riding with $U_{\text {iso }}(\mathrm{H})=1.2 U_{\text {eq }}$ or 1.5 Ueq.

\section{Discussion}

In the title crystal structure copper(II) atom is chelated by one unsymmetrical Schiff base ligand providing their amide, amine $\mathrm{N}$ atoms and phenolate $\mathrm{O}$ atom and one water molecule (figure, top). A distorted square planar $\mathrm{CuN}_{2} \mathrm{O}_{2}$ environment arises with the $\mathrm{N} 1 \cdots \mathrm{N} 2$ and $\mathrm{O} 1 \cdots \mathrm{O} 4$ distances $(2.605 \AA$ and $2.662 \AA$, respectively) shorter than the $\mathrm{N} 1 \cdots \mathrm{O} 1$ and $\mathrm{N} 2 \cdots \mathrm{O} 4$ distances $(2.801 \AA$ and $2.902 \AA$, respectively). The $\mathrm{Cu} 1-\mathrm{N} 1$ distance $(1.923 \AA)$ is shorter than $\mathrm{Cu} 1-\mathrm{N} 2(1.969 \AA)$ suggesting that the imine nitrogen atom is a slightly better donor than amide nitrogen atom because the electron density is delocalized into the sulfonyl group. The $\mathrm{Cu} 1-\mathrm{O} 4$ (water) distance is longer than $\mathrm{Cu} 1-\mathrm{O} 1$ (phenolate). The four coordinated atoms $\left(\mathrm{N}_{2} \mathrm{O}_{2}\right)$ are arranged in a cisconformation. In the molecule, the chelated five-membered ring (Cu1-N1-C8-C9-N2) and six-membered ring (Cu1-O1-C2-C1C7-N1) also contribute to the stability of the complex. The bondvalence sum for the $\mathrm{Cu}$ center is calculated to be 2.2 , which is close to the expected value of 2 [1].

A strong intramolecular $\mathrm{O}-\mathrm{H} \cdots \mathrm{O}$ hydrogen bond helps to stabilize the molecular conformation $(\mathrm{O} 4-\mathrm{H} 4 \mathrm{~B} \cdots \mathrm{O} 2$ with $0.66(7) \AA$, 2.02(7) $\left.\AA, 2.603(3) \AA, 149(8)^{\circ}\right)$. The resulting six-membered chelate ring $(\mathrm{Cu} 1-\mathrm{N} 2-\mathrm{S} 1-\mathrm{O} 2 \cdots \mathrm{H} 4 \mathrm{~B}-\mathrm{O} 4)$ is planar with a mean deviation of $0.0830 \AA$. The crystal packing may be further stabilized by $\mathrm{C}-\mathrm{H} \cdots \mathrm{O}, \mathrm{C}-\mathrm{H} \cdots \pi$ and $\pi \cdots \pi$ interactions (figure, middle). The resulting $\mathrm{C} 12-\mathrm{H} 12 \cdots \mathrm{O} 3$ hydrogen bonds among different symmetric motifs link the structure in layers running parallel to the $a$ axis and shows a centrosymmetric arrangement mode. In the structure, there are the two $\pi \cdots \pi$ interactions formed between two rings of one molecule and its symmetry equivalent by inversion $(-x,-y,-z)$. The $\mathrm{Cg} \cdots \mathrm{Cg}^{\mathrm{i}}$ distance is $3.515 \AA$, where $\mathrm{Cg}$ and $\mathrm{Cg}^{\mathrm{i}}$ are the centroids of ring 1 (Cu1-O1-C2-C1-C7-N1) and ring 2 (C10 
to C15) (figure, middle). Two molecules are arranged in a headto-tail mode and thus formed a subunit. The subunits are linked by hydrogen bonding and form two-dimensional layers parallel to the $b$ axis (figure, bottom).

Table 1. Data collection and handling.

\begin{tabular}{ll}
\hline Crystal: & dark green plate, \\
& size $0.11 \times 0.27 \times 0.38 \mathrm{~mm}$ \\
Wavelength: & Mo $K_{\alpha}$ radiation $(0.71073 \AA)$ \\
$\mu:$ & $14.97 \mathrm{~cm}^{-1}$ \\
Diffractometer, scan mode: & Bruker SMART CCD, $\varphi / \omega$ \\
$2 \theta_{\text {max }}:$ & $50.18^{\circ}$ \\
$N(h k l)_{\text {measured }}, N(h k l)_{\text {unique }}:$ & 4093,2820 \\
Criterion for $I_{\mathrm{obs}}, N(h k l)_{\mathrm{gt}}:$ & $I_{\mathrm{obs}}>2 \sigma\left(I_{\mathrm{obs}}\right), 2385$ \\
$N(\text { param })_{\text {refined }}$ & 195 \\
Programs: & SHELXS-97 [2], SHELXL-97 [3], \\
& SHELXTL [4] \\
\hline
\end{tabular}

Table 2. Atomic coordinates and displacement parameters (in $\AA^{2}$ ).

\begin{tabular}{llllll}
\hline Atom & Site & $x$ & $y$ & $z$ & $U_{\text {iso }}$ \\
\hline $\mathrm{H}(11)$ & $2 i$ & 0.3834 & 0.2480 & 0.3027 & 0.069 \\
$\mathrm{H}(12)$ & $2 i$ & 0.3617 & 0.4434 & 0.4075 & 0.077 \\
$\mathrm{H}(14)$ & $2 i$ & 0.1752 & 0.1121 & 0.5723 & 0.063 \\
$\mathrm{H}(15)$ & $2 i$ & 0.1969 & -0.0834 & 0.4676 & 0.053 \\
$\mathrm{H}(3)$ & $2 i$ & 0.1725 & 0.0948 & -0.1500 & 0.076 \\
$\mathrm{H}(4)$ & $2 i$ & -0.0726 & 0.3202 & -0.2260 & 0.086 \\
$\mathrm{H}(5)$ & $2 i$ & -0.3580 & 0.5904 & -0.1642 & 0.088 \\
$\mathrm{H}(6)$ & $2 i$ & -0.4151 & 0.6325 & -0.0259 & 0.069 \\
$\mathrm{H}(7)$ & $2 i$ & -0.3328 & 0.5249 & 0.1052 & 0.051 \\
$\mathrm{H}(8 \mathrm{~A})$ & $2 i$ & -0.3192 & 0.4028 & 0.2393 & 0.051 \\
$\mathrm{H}(8 \mathrm{~B})$ & $2 i$ & -0.1374 & 0.4528 & 0.2432 & 0.051 \\
$\mathrm{H}(9 \mathrm{~A})$ & $2 i$ & -0.0815 & 0.1841 & 0.3309 & 0.051 \\
$\mathrm{H}(9 \mathrm{~B})$ & $2 i$ & -0.1256 & 0.0700 & 0.2683 & 0.051 \\
$\mathrm{H}(16 \mathrm{~A})$ & $2 i$ & 0.3772 & 0.3704 & 0.5892 & 0.123 \\
$\mathrm{H}(16 \mathrm{~B})$ & $2 i$ & 0.1423 & 0.4306 & 0.6124 & 0.123 \\
$\mathrm{H}(16 \mathrm{C})$ & $2 i$ & 0.2170 & 0.5592 & 0.5484 & 0.123 \\
$\mathrm{H}(4 \mathrm{~A})$ & $2 i$ & $0.513(8)$ & $0.037(8)$ & $0.106(3)$ & 0.123 \\
$\mathrm{H}(4 \mathrm{~B})$ & $2 i$ & $0.520(9)$ & $-0.108(9)$ & $0.126(4)$ & 0.123 \\
\end{tabular}

Table 3. Atomic coordinates and displacement parameters (in $\AA^{2}$ ).

\begin{tabular}{|c|c|c|c|c|c|c|c|c|c|c|}
\hline Atom & Site & $x$ & $y$ & $z$ & $U_{11}$ & $U_{22}$ & $U_{33}$ & $U_{12}$ & $U_{13}$ & $U_{23}$ \\
\hline$C(10)$ & $2 i$ & $0.2923(2)$ & $0.0629(2)$ & $0.37477(6)$ & $0.036(1)$ & $0.038(2)$ & $0.029(1)$ & $-0.012(1)$ & $-0.006(1)$ & $0.005(1)$ \\
\hline $\mathrm{C}(11)$ & $2 i$ & $0.3417(2)$ & $0.2207(2)$ & $0.35676(6)$ & $0.074(2)$ & $0.061(2)$ & $0.044(2)$ & $-0.037(2)$ & $-0.013(2)$ & $0.016(2)$ \\
\hline$C(12)$ & $2 i$ & $0.3287(3)$ & $0.3378(2)$ & $0.41951(8)$ & $0.085(3)$ & $0.050(2)$ & $0.072(3)$ & $-0.038(2)$ & $-0.033(2)$ & $0.014(2)$ \\
\hline$C(13)$ & $2 i$ & $0.2663(3)$ & $0.2971(2)$ & $0.50027(7)$ & $0.046(2)$ & $0.045(2)$ & $0.060(2)$ & $-0.007(1)$ & $-0.024(2)$ & $-0.008(2)$ \\
\hline$C(14)$ & $2 i$ & $0.2170(2)$ & $0.1393(2)$ & $0.51828(6)$ & $0.050(2)$ & $0.068(2)$ & $0.031(2)$ & $-0.022(2)$ & $-0.001(1)$ & $-0.005(1)$ \\
\hline$C(15)$ & $2 i$ & $0.2300(2)$ & $0.0222(2)$ & $0.45553(6)$ & $0.048(2)$ & $0.052(2)$ & $0.033(2)$ & $-0.025(1)$ & $-0.004(1)$ & $0.003(1)$ \\
\hline $\mathrm{C}(2)$ & $2 i$ & $0.0290(2)$ & $0.2335(2)$ & $-0.03873(6)$ & $0.071(2)$ & $0.053(2)$ & $0.032(2)$ & $-0.043(2)$ & $-0.013(1)$ & $0.004(1)$ \\
\hline$C(3)$ & $2 i$ & $0.0572(3)$ & $0.2032(3)$ & $-0.12464(6)$ & $0.107(3)$ & $0.073(2)$ & $0.035(2)$ & $-0.062(2)$ & $-0.014(2)$ & $0.002(2)$ \\
\hline$C(4)$ & $2 i$ & $-0.0906(3)$ & $0.3383(3)$ & $-0.16992(7)$ & $0.117(3)$ & $0.110(3)$ & $0.038(2)$ & $-0.090(3)$ & $-0.029(2)$ & $0.017(2)$ \\
\hline$C(5)$ & $2 i$ & $-0.2636(3)$ & $0.4991(3)$ & $-0.13266(8)$ & $0.087(3)$ & $0.122(4)$ & $0.052(2)$ & $-0.079(3)$ & $-0.039(2)$ & $0.040(2)$ \\
\hline$C(6)$ & $2 i$ & $-0.2970(3)$ & $0.5248(3)$ & $-0.05015(8)$ & $0.055(2)$ & $0.087(3)$ & $0.052(2)$ & $-0.048(2)$ & $-0.023(2)$ & $0.026(2)$ \\
\hline $\mathrm{Cu}(1)$ & $2 i$ & $0.17992(5)$ & $0.10221(5)$ & $0.11628(2)$ & $0.0501(2)$ & $0.0365(2)$ & $0.0250(2)$ & $-0.0057(2)$ & $-0.0030(2)$ & $-0.0019(1)$ \\
\hline $\mathrm{S}(1)$ & $2 i$ & $0.3030(1)$ & $-0.0811(1)$ & $0.29415(4)$ & $0.0559(5)$ & $0.0359(4)$ & $0.0276(4)$ & $-0.0059(3)$ & $-0.0075(3)$ & $0.0008(3)$ \\
\hline $\mathrm{O}(1)$ & $2 i$ & $0.1808(4)$ & $0.1086(3)$ & $0.0012(1)$ & $0.075(2)$ & $0.045(1)$ & $0.028(1)$ & $-0.020(1)$ & $-0.006(1)$ & $-0.0025(9)$ \\
\hline $\mathrm{O}(3)$ & $2 i$ & $0.2434(4)$ & $-0.2299(3)$ & $0.3324(1)$ & $0.114(2)$ & $0.042(1)$ & $0.049(1)$ & $-0.035(1)$ & $-0.028(1)$ & $0.010(1)$ \\
\hline $\mathrm{O}(4)$ & $2 i$ & $0.4714(5)$ & $-0.0780(5)$ & $0.0944(2)$ & $0.069(2)$ & $0.098(2)$ & $0.039(2)$ & $0.026(2)$ & $0.001(1)$ & $0.011(2)$ \\
\hline $\mathrm{N}(1)$ & $2 i$ & $-0.0978(3)$ & $0.3098(3)$ & $0.1392(1)$ & $0.040(1)$ & $0.040(1)$ & $0.031(1)$ & $-0.018(1)$ & $-0.007(1)$ & $0.003(1)$ \\
\hline $\mathrm{N}(2)$ & $2 i$ & $0.1510(4)$ & $0.0682(4)$ & $0.2362(1)$ & $0.046(1)$ & $0.042(1)$ & $0.026(1)$ & $-0.008(1)$ & $-0.004(1)$ & $0.001(1)$ \\
\hline$C(7)$ & $2 i$ & $-0.2074(4)$ & $0.4181(5)$ & $0.0860(2)$ & $0.037(2)$ & $0.048(2)$ & $0.044(2)$ & $-0.022(1)$ & $-0.010(1)$ & $0.008(1)$ \\
\hline $\mathrm{C}(8)$ & $2 i$ & $-0.1714(4)$ & $0.3527(4)$ & $0.2272(2)$ & $0.038(2)$ & $0.048(2)$ & $0.033(2)$ & $-0.014(1)$ & $-0.003(1)$ & $-0.003(1)$ \\
\hline $\mathrm{C}(9)$ & $2 i$ & $-0.0663(4)$ & $0.1585(4)$ & $0.2733(2)$ & $0.049(2)$ & $0.046(2)$ & $0.030(2)$ & $-0.021(1)$ & $-0.003(1)$ & $-0.001(1)$ \\
\hline$C(16)$ & $2 i$ & $0.2491(6)$ & $0.4262(6)$ & $0.5689(3)$ & $0.075(3)$ & $0.066(2)$ & $0.092(3)$ & $-0.007(2)$ & $-0.036(2)$ & $-0.029(2)$ \\
\hline
\end{tabular}

Acknowledgment. We thank the Shaanxi Normal University for the generous support of this study.

\section{References}

1. Thorp, H. H.: Bond valence sum analysis of metal-ligand bond lengths in metalloenzymes and model complexes. Inorg. Chem. 31 (1992) 1585-1588.

2. Sheldrick, G. M.: SHELXS-97. Program for the Solution of Crystal Structures. University of Göttingen, Germany 1997.
3. Sheldrick, G. M.: SHELXL-97. Program for the Refinement of Crystal Structures. University of Göttingen, Germany 1997.

4. Sheldrick, G. M.: SHELXTL. Structure Determination Software Suite. Version 5. Bruker AXS, Madison, Wisconsin, USA 2001. 